\title{
A Noniterative Method for the Generation of Orthogonal Coordinates in Doubly-Connected Regions*
}

\author{
By Z. U. A. Warsi and J. F. Thompson
}

\begin{abstract}
In this paper a noniterative method for the numerical generation of orthogonal curvilinear coordinates for plane annular regions between two arbitrary smooth closed curves has been developed. The basic generating equation is the Gaussian equation for a Euclidean space under the constraint of orthogonality. The resulting equation has been solved analytically for the case of isothermic coordinates. A coordinate transformation then yields nonisothermic coordinates in which the outgoing coordinate can be distributed in any desired manner. The method has been applied in many cases and these test results demonstrate that the proposed method can be readily applied in a variety of problems.
\end{abstract}

1. Introduction. The problem of generating orthogonal or nonorthogonal curvilinear coordinates $\xi, \eta$ in arbitrary curvilinear domains is a problem of current interest in fluid mechanics and other branches of physics and engineering. The idea of generating coordinate meshes by numerically solving a set of partial differential equations under the boundary-geometric data as the boundary conditions arose with the work of Winslow [24]. Later Barfield [2], Chu [4], Godunov and Prokopov [10], Amsden and Hirt [1], and Potter and Tuttle [17] used this concept in generating coordinate curves for specific physical situations. The whole concept has, however, been used in a much organized manner by Thompson, Thames and Mastin [20] in developing and coding [21] the computer program for generating nonorthogonal coordinates in a variety of two-dimensional situations. The user, however, has no control over the orthogonality or nonorthogonality of the generated coordinates.

The underlying basis of all the above methods, including that of Pope [16], Starius [19], Middlecoff and Thomas [14], and Mobley and Stewart [15] is the choice of a set of coupled partial differential equations. Two exceptions to the above are the methods of Eiseman [7], whose method is of an algebraic-geometric nature, and that of Davis [6], which is based on the Schwarz-Christoffel transformation of the complex variable theory.

In the differential equations method, except for the work of Starius [19] where a hyperbolic system of equations is used, all other methods rest on the system of elliptic partial differential equations of the Laplace form. These equations are in

Received November 10, 1980.

1980 Mathematics Subject Classification. Primary 14D99, 31A05, 30C60.

Key words and phrases. Grid generation, mappings, potential theory.

* This work has been supported in part by the Air Force Office of Scientific Research under Grants AFOSR No. 76-2922 and No. 80-0185. 
essence a set of arbitrary differential constraints on the distributions of the fundamental metric coefficients $g_{i j}$.

In this paper we develop a new approach by providing another differential constraint on the $g_{i j}$ 's which is not based on any arbitrary assumption. This relationship is already available to hand by the condition that the coordinates are to be generated in a Euclidean space. The most natural choice is then to use the Gaussian equation [8] for a Euclidean space, viz., a space of zero curvature. This fundamental equation is one equation in three unknowns, $g_{11}, g_{12}$ and $g_{22}$, which becomes a deterministic equation in one unknown only in the case of isothermic coordinates, i.e., $g_{12}=0$ and $g_{22}=g_{11}$. This equation has been solved in an exact fashion. Further, from the earlier work of Potter and Tuttle [17], we have the result that in the case of orthogonal coordinates the ratio $g_{11} / g_{22}$ can be taken as a product of functions of $\xi$ and $\eta$. This result has been used to show that the result obtained for isothermic coordinates $\left(g_{22}=g_{11}\right)$ can be used to provide the solution for nonisothermic orthogonal coordinates $\left(g_{22} \neq g_{11}\right)$ by a coordinate transformation. This coordinate transformation is dependent on the user's choice, that is, how one wants to redistribute the coordinates in a given region.

The method developed on the preceding ideas therefore provides a noniterative closed form analytic solution for the case of two-dimensional orthogonal coordinates. Most of the numerical work is in the numerical quadratures and summation of a truncated Fourier expansion. Some test result cases are demonstrated in Figures $3-8,[22]$.

2. Formulation of the Problem. All methods of numerical coordinate generation in a two-dimensional plane and classified under the method of "elliptic equations" ([20], [21]) have depended invariably on the solution of the Poisson equations for the curvilinear coordinates $\xi(x, y)$ and $\eta(x, y)$ which may be given the forms

$$
\begin{aligned}
& \nabla^{2} \xi=\frac{-g_{22}}{g} P(\xi, \eta), \\
& \nabla^{2} \eta=\frac{-g_{11}}{g} Q(\xi, \eta),
\end{aligned}
$$

where $P(\xi, \eta)$ and $Q(\xi, \eta)$ are arbitrarily specified control functions, and $g_{i j}$ are the fundamental metric coefficients. Equation (1) can also be written in the following forms:

$$
\begin{aligned}
& g_{11} \Gamma_{22}^{1}-2 g_{12} \Gamma_{12}^{1}+g_{22} \Gamma_{11}^{1}=g_{22} P(\xi, \eta), \\
& g_{11} \Gamma_{22}^{2}-2 g_{12} \Gamma_{12}^{2}+g_{22} \Gamma_{11}^{2}=g_{11} Q(\xi, \eta),
\end{aligned}
$$

where $\Gamma_{j k}^{i}$ are the Christoffel symbols of the second kind

$$
\begin{gathered}
\Gamma_{j k}^{i}=g^{i l}[j k, l], \\
{[j k, l]=\frac{1}{2}\left(\frac{\partial g_{j l}}{\partial x^{k}}+\frac{\partial g_{k l}}{\partial x^{j}}-\frac{\partial g_{j k}}{\partial x^{l}}\right),} \\
g=g_{11} g_{22}-\left(g_{12}\right)^{2} .
\end{gathered}
$$

From (1) and (2) we conclude that by choosing $P$ and $Q$ arbitrarily (which can also be set equal to zero), one specifies a constraint between the $g_{i j}$ and their first 
partial derivatives. The choice of (1) as a set of generating equations is due to the fact that these equations are the simplest elliptic equations satisfying an extremum principle. Besides the simplicity of their forms and the ease with which these equations or their inverted forms can be solved on a computer, it remains a fact that the coordinates in two dimensions always satisfy a set of Laplace-form of equations no matter which other equations or methods are used to generate them. The appearance of $P$ and $Q$ in (1) cloud this fact as they are left completely unspecified. To clarify this point we have inserted (2) to show that the specification of $P$ and $Q$ amounts to specifying a set of differential constraints on the $g_{i j}$ 's. This automatically raises a question: is there a differential constraint on the $g_{i j}$ 's which must always be satisfied no matter what method is used to generate the coordinates or what $P$ and $Q$ are? In essence, the method developed in this paper is based on an equation which is obtained by addressing the above question.

Since the purpose is to generate curvilinear coordinates in a plane, we consider the equation of Gauss which for zero curvature is, (cf. [8]),

$$
\frac{\partial}{\partial \eta}\left(\frac{\sqrt{g} \Gamma_{11}^{2}}{g_{11}}\right)-\frac{\partial}{\partial \xi}\left(\frac{\sqrt{g} \Gamma_{12}^{2}}{g_{11}}\right)=0 .
$$

Equation (3) is the fundamental equation which must always be satisfied by the metric coefficient $g_{i j}$ in a plane. Below we proceed with the consequences of this equation and then particularize it for the case of orthogonal coordinates.

Equation (3) implies the existence of a function $\alpha(\xi, \eta)$ which is commutative in cross derivatives, i.e., $\alpha_{\eta \xi}=\alpha_{\xi \eta}$. Thus (3) is automatically satisfied by the equations

$$
\alpha_{\xi}=\frac{-\sqrt{g}}{g_{11}} \Gamma_{11}^{2}, \quad \alpha_{\eta}=\frac{-\sqrt{g}}{g_{11}} \Gamma_{12}^{2} .
$$

Specifically, $\alpha$ is the angle of inclination with respect to the $x$-axis of the tangent to the coordinate line $\eta=$ const directed in the sense of increasing values of the parameter $\xi$. In terms of $\alpha$, the first partial derivatives of $x$ and $y$ are

$$
\begin{aligned}
& x_{\xi}=\sqrt{g_{11}} \cos \alpha, \quad y_{\xi}=-\sqrt{g_{11}} \sin \alpha, \\
& x_{\eta}=\frac{1}{\sqrt{g_{11}}}\left(g_{12} \cos \alpha+\sqrt{g} \sin \alpha\right), \\
& y_{\eta}=\frac{1}{\sqrt{g_{11}}}\left(\sqrt{g} \cos \alpha-g_{12} \sin \alpha\right),
\end{aligned}
$$

and thus $x$ and $y$ are given by the line integrals

$$
\begin{aligned}
& x=\int\left[\sqrt{g_{11}} \cos \alpha d \xi+\frac{1}{\sqrt{g_{11}}}\left(g_{12} \cos \alpha+\sqrt{g} \sin \alpha\right) d \eta\right] \\
& y=-\int\left[\sqrt{g_{11}} \sin \alpha d \xi-\frac{1}{\sqrt{g_{11}}}\left(\sqrt{g} \cos \alpha-g_{12} \sin \alpha\right) d \eta\right] .
\end{aligned}
$$


We note in passing that the inverse relations to (5) are

$$
\begin{gathered}
\xi_{x}=\left(\sqrt{g} \cos \alpha-g_{12} \sin \alpha\right) / \sqrt{g g_{11}}, \\
\xi_{y}=-\left(g_{12} \cos \alpha+\sqrt{g} \sin \alpha\right) / \sqrt{g g_{11}}, \\
\eta_{x}=\sqrt{g_{11} / g} \sin \alpha, \\
\eta_{y}=\sqrt{g_{11} / g} \cos \alpha .
\end{gathered}
$$

From (4), we also find that

$$
\alpha=-\int \frac{\sqrt{g}}{g_{11}}\left(\Gamma_{11}^{2} d \xi+\Gamma_{12}^{2} d \eta\right) .
$$

The expressions from (5)-(8) imply that if the metric coefficients $g_{i j}$ are known by some means, then the Cartesian coordinates $x$ and $y$, the angle $\alpha$, and all the partial derivatives of $x$ and $y$ can be obtained in a simple way.

The differential constraint provided by (3) is a quasilinear partial differential equation involving all the three metric coefficients; $g_{11}, g_{12}$ and $g_{22}$. For the evaluation of these three unknowns, one needs to provide two more differential or algebraic relations between these coefficients. It will be seen shortly that in the case of isothermic coordinates, (3) provides a deterministic equation in a single metric coefficient.

For orthogonal coordinates,

$$
g_{12}=x_{\xi} x_{\eta}+y_{\xi} y_{\eta}=0
$$

so that there exists a function $F>0$ such that

$$
x_{\eta}=-F y_{\xi}, \quad y_{\eta}=F x_{\xi} .
$$

Substituting (9) in (3), we obtain the equation

$$
\frac{\partial}{\partial \xi}\left[\frac{1}{F g_{11}} \frac{\partial}{\partial \xi}\left(F^{2} g_{11}\right)\right]+\frac{\partial}{\partial \eta}\left(\frac{1}{F g_{11}} \frac{\partial g_{11}}{\partial \eta}\right)=0
$$

where

$$
F^{2}=g_{22} / g_{11}
$$

and

$$
g=\left(F g_{11}\right)^{2}
$$

2.1 Case of Isothermic Coordinates. Isothermic coordinates $\xi$ and $\eta$ are such that in these coordinates the length element $d s$ is given by

$$
(d s)^{2}=\lambda^{2}\left(d \xi^{2}+d \eta^{2}\right) .
$$

Thus, in isothermic coordinates,

$$
g_{12}=0, \quad g_{22}=g_{11}=\left(\lambda^{2}\right),
$$

so that $F=1$, and (11) becomes a simple linear equation

$$
\frac{\partial^{2} P}{\partial \xi^{2}}+\frac{\partial^{2} P}{\partial \eta^{2}}=0
$$


where

$$
P=\ln \left(g_{11}\right) \text {. }
$$

Thus the Gauss equation for a plane reduces to an equation of the Laplace form in $P$ when the coordinates $\xi$ and $\eta$ are isothermic.
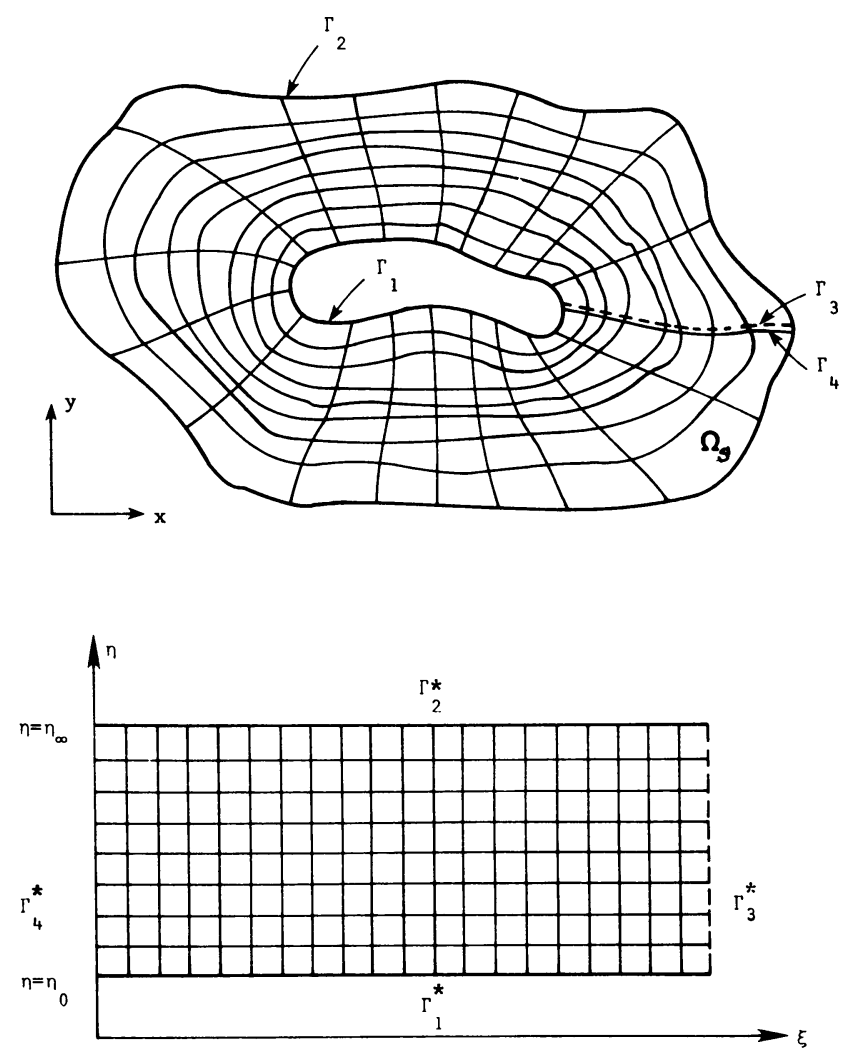

$$
\begin{gathered}
\text { Transformed Plane } \\
\text { (Natural Coordinates) }
\end{gathered}
$$

FIGURE 1

\section{Physical and transformed planes}

We now pose the problem of generating isothermic coordinates between an annulus bounded by two arbitrary smooth closed curves. Referring to Figure 1, let the boundary $\Gamma_{2}$ of a bounded region $\Omega$ in a Euclidean two-dimensional space be a simple closed curve $x=x_{\infty}(\xi), y=y_{\infty}(\xi)$, with a uniformly turning tangent. In the region $\Omega$, let $\Omega_{s}$ be an annular subregion bounded by the inner boundary $\Gamma_{1}$ and the outer boundary $\Gamma_{2}$. The region $\Omega_{s}$ is to be mapped onto a rectangular region $R$ in the $\xi \eta$-plane so as to have

$$
\left.\begin{array}{l}
x=x(\xi, \eta) \\
y=y(\xi, \eta)
\end{array}\right\}, \quad \eta_{\beta} \leqslant \eta \leqslant \eta_{\infty}
$$

where $x$ and $y$ are periodic in the $\xi$-argument, and $\eta_{\beta}, \eta_{\infty}$ are the actual parametric values associated with the inner and outer boundaries, respectively. We now assume 
that we have established a method of enumeration in the $\xi$-parameter which is such that there is an orthogonal correspondence between the same $\xi$-points on the inner and outer boundaries; see Section 2.2. Based on this $\xi$-distribution, we easily pick out the values of $x$ and $y$ and so by differentiation the values of $P$. Thus the boundary conditions for (14) are

$$
\left.\begin{array}{rl}
P & =P_{\beta}(\xi) \quad \text { at } \eta=0^{* *} \\
& =P_{\infty}(\xi) \quad \text { at } \eta=\eta_{\beta}
\end{array}\right\}, \quad 0 \leqslant \xi \leqslant 2 \pi,
$$

where the subscripts $\beta$ and $\infty$ denote the inner and outer boundaries, respectively. The periodicity requirement is that

$$
P(\xi, \eta)=P(\xi+2 \pi, \eta)
$$

A general analytic solution of (14) under the boundary and periodicity conditions (17) and (18) is

$$
\begin{aligned}
P(\xi, \eta)= & a_{0}+\eta \bar{K}+\sum_{n=1}^{\infty}\left(a_{n} \cos n \xi+b_{n} \sin \xi\right) \sinh n\left(\eta_{\infty}-\eta\right) / \sinh n \eta_{\infty} \\
& +\sum_{n=1}^{\infty}\left(c_{n} \cos n \xi+d_{n} \sin n \xi\right) \sinh n \eta / \sinh n \eta_{\infty}
\end{aligned}
$$

where

$$
\bar{K}=\left(c_{0}-a_{0}\right) / \eta_{\infty}
$$

and

$$
\begin{gathered}
a_{0}=\frac{1}{2 \pi} \int_{0}^{2 \pi} P_{\beta}(\xi) d \xi, \quad c_{0}=\frac{1}{2 \pi} \int_{0}^{2 \pi} P_{\infty}(\xi) d \xi \\
a_{n}=\frac{1}{\pi} \int_{0}^{2 \pi} P_{\beta}(\xi) \cos n \xi d \xi, \quad b_{n}=\frac{1}{\pi} \int_{0}^{2 \pi} P_{\beta}(\xi) \sin n \xi d \xi \\
c_{n}=\frac{1}{\pi} \int_{0}^{2 \pi} P_{\infty}(\xi) \cos n \xi d \xi, \quad d_{n}=\frac{1}{\pi} \int_{0}^{2 \pi} P_{\infty}(\xi) \sin n \xi d \xi
\end{gathered}
$$

Since $P_{\beta}(\xi)$ and $P_{\infty}(\xi)$ are prescribed functions, the values of the coefficients $a_{0}, \ldots, d_{n}$ can be obtained by numerical quadratures.

For orthogonal coordinates,

$$
\alpha_{\xi}=\frac{1}{2 \sqrt{g}} \frac{\partial g_{11}}{\partial \eta}, \quad \alpha_{\eta}=-\frac{1}{2 \sqrt{g}} \frac{\partial g_{22}}{\partial \xi},
$$

so that, for the case of isothermic coordinates $\left(g_{22}=g_{11}\right)$, we have

$$
\alpha_{\xi}=\frac{1}{2} \frac{\partial P}{\partial \eta}, \quad \alpha_{\eta}=-\frac{1}{2} \frac{\partial P}{\partial \xi}
$$

** There is no loss of generality in setting the parametric value $\eta_{\beta}=0$. The value $\eta_{\infty}$ must be interpreted as the difference between the actual values of $\eta$ at the outer and inner boundaries. 
Using the expression for $P$ given in (19), we obtain an exact expression for $\alpha(\xi, \eta)$ by integration as

$$
\begin{aligned}
\alpha(\xi, \eta)= & \alpha(\xi, 0)+\sum_{n=1}^{\infty} \frac{\cosh n\left(\eta_{\infty}-\eta\right)}{2 \sinh n \eta_{\infty}}\left(b_{n} \cos n \xi-a_{n} \sin n \xi\right) \\
& +\sum_{n=1}^{\infty} \frac{\cosh n \eta}{2 \sinh n \eta_{\infty}}\left(c_{n} \sin n \xi-d_{n} \cos n \xi\right) \\
& -\sum_{n=1}^{\infty} \frac{\cosh n \eta_{\infty}}{2 \sinh n \eta_{\infty}}\left(b_{n} \cos n \xi-a_{n} \sin n \xi\right) \\
& -\sum_{n=1}^{\infty} \frac{1}{2 \sinh n \eta_{\infty}}\left(c_{n} \sin n \xi-d_{n} \cos n \xi\right) .
\end{aligned}
$$

Having determined $g_{11}=\exp (P(\xi, \eta))$ and $\alpha(\xi, \eta)$, we can find the Cartesian coordinates

$$
\begin{aligned}
& x(\xi, \eta)=x(\xi, 0)+\int_{0}^{\eta} \sqrt{g_{22}} \sin \alpha d \eta, \\
& y(\xi, \eta)=y(\xi, 0)+\int_{0}^{\eta} \sqrt{g_{22}} \cos \alpha d \eta,
\end{aligned}
$$

where, according to the foregoing analysis $g_{22}=g_{11}$.

The preceding analysis thus completes the solution technique for isothermic coordinates in an annulus bounded by arbitrary closed curves.

2.2 Choice of $\xi$-Points on Boundary Curves. As noted earlier (cf. discussion after Eq. (16)), the choice of those $\xi$-points on the inner and outer boundary curves which are in orthogonal correspondence is crucial to the success of the method. In this connection we note that for isothermic coordinates, from (7) by cross differentiation, the usual condition equations

$$
\nabla^{2} \xi=0, \quad \nabla^{2} \eta=0
$$

are available. These equations, besides being satisfied in the field, must also be satisfied at the boundaries of the annulus. The second equation in (25) has the meaning that each coordinate $\eta$-const, including the boundaries, must be free from sources or sinks. Since the first equation in (25) is implicitly satisfied in the field by the solution of (14), we have only to choose the $\xi$-points on the boundaries so that it is again satisfied.

We now propose an indirect method so that the equation

$$
\nabla^{2} \xi=0
$$

is satisfied for the boundary points. To achieve this, we note that in many cases it is possible to circumscribe circles around both the inner and outer boundaries. We now consider the conformal transformation of the annulus between these circular boundaries in the physical plane (the plane of curves $\beta$ and $\infty$ or $\Gamma_{1}$ and $\Gamma_{2}$ ) onto the annulus between the concentric circles in the transformed plane and take the coordinate $\xi$ as the angle traced out in a clockwise sense by the common radii in the transformed plane. Now two cases arise depending on whether the circumscribed 
circles are concentric or not as shown in Figure 2. If the circumscribed circles are concentric, then their conformal transformation will again be concentric circles without any change in the ratio of the radii in the two planes [11, p. 208]. Thus in this case we select those values of the ordinates on $\Gamma_{1}$ and $\Gamma_{2}$ which correspond to the abscissae

$$
x_{\beta}=r_{S} \cos \xi, \quad x_{\infty}=r_{L} \cos \xi,
$$

where $r_{S}$ and $r_{L}$ are the radii of the inner and outer circles circumscribing $\Gamma_{1}$ and $\Gamma_{2}$ respectively.

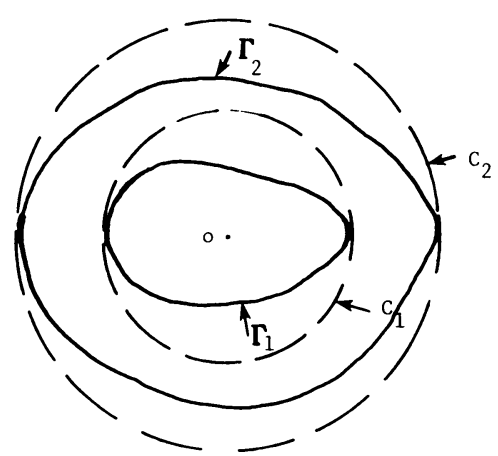

(a)
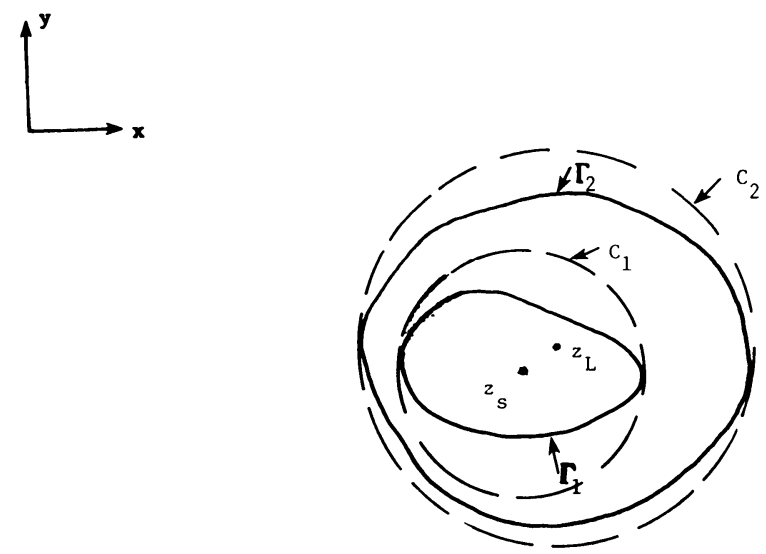

(b)

FIGURE 2

(a) Concentric circumscribed circles $C_{1}$ and $C_{2}$ of radii $r_{S}, r_{L}$ respectively with center at the origin. (b) Nonconcentric circumscribed circles $C_{1}$ and $C_{2}$ of radii $r_{S}$ and $r_{L}$ and centers at $z_{S}$ and $z_{L}$ respectively

If the circumscribed circles are nonconcentric (Figure (2b)), then using the conformal transformation of nonconcentric to concentric circles, Kober [13], we select those values of the ordinates on $\Gamma_{1}$ and $\Gamma_{2}$ which correspond to the abscissae 
given by the following expression. In this expression the quantity $\gamma$ and other quantities are

$$
\begin{gathered}
\gamma=1 \text { for the outer boundary } \Gamma_{2}, \\
\gamma=\frac{r_{L}}{r_{S}}\left|\frac{d-t}{t}\right| \text { for the inner boundary } \Gamma_{1}, \\
d^{2}=\left(x_{S}-x_{L}\right)^{2}+\left(y_{S}-y_{L}\right)^{2}, \\
\left(x_{S}, y_{S}\right)=\text { center of the inner circle, } \\
\left(x_{L}, y_{L}\right)=\text { center of the outer circle, } \\
r_{S}, r_{L}=\text { radii of the inner and outer circle, respectively, } \\
c=\left[\left(d^{2}+r_{L}^{2}-r_{S}^{2}\right)+\left\{\left(d^{2}+r_{L}^{2}-r_{S}^{2}\right)^{2}-4 d^{2} r_{L}^{2}\right\}^{1 / 2}\right] / 2 r_{L} d, \\
\Psi=\pi-\tan { }^{-1}\left(\frac{y_{S}-y_{L}}{x_{S}-x_{L}}\right), \\
x(\xi)=\left[( 1 - c \gamma \operatorname { c o s } \xi ) \left\{x_{L}(1-c \gamma \cos \xi)+c \gamma y_{L} \sin \xi\right.\right. \\
\left.+r_{L}(c \cos \Psi-\gamma \cos (\xi-\Psi))\right\} \\
-c \gamma\left\{y_{L}(1-c \gamma \cos \xi)-c \gamma x_{L} \sin \xi\right. \\
\left.\left.-r_{L}(c \sin \Psi+\gamma \sin (\xi-\Psi))\right\} \sin \xi\right] /\left(1-2 c \gamma \cos \xi+c^{2} \gamma^{2}\right) .
\end{gathered}
$$

Having determined the appropriate sets $\left(x_{\beta}(\xi), y_{\beta}(\xi)\right)$ and $\left(x_{\infty}(\xi), y_{\infty}(\xi)\right)$, we now calculate the radius ratios by using the formula

$$
\rho_{0}=\frac{1}{2 \pi a} \int_{0}^{2 \pi}\left[x_{\infty}(\xi) \cos \xi-y_{\infty}(\xi) \sin \xi\right] d \xi
$$

where

$$
a=\frac{1}{2 \pi} \int_{0}^{2 \pi}\left[x_{\beta}(\xi) \cos \xi-y_{\beta}(\xi) \sin \xi\right] d \xi
$$

These terms are obtained by the first term of the Laurent series as shown in [18].

The parametric difference $\eta_{\infty}$ is connected in some manner with the "modulus" of the domain which, however, by itself is a separate problem (see, e.g., Burbea [3] and Gaier [9]). In this work we have defined $\eta_{\infty}$ based on the knowledge of the radius ratio $\rho_{0}$ through the simple formula

$$
\eta_{\infty}=\ln \rho_{0} .
$$

2.3 Case of Nonisothermic Coordinates. We now consider the case of orthogonal coordinates $\xi$ and $\eta$ when they are nonisothermic. In this case the element of length in the plane is given by

$$
(d s)^{2}=g_{11} d \xi^{2}+g_{22} d \eta^{2}, \quad g_{22} \neq g_{11} .
$$

The governing equation for such a case is of course (11). Our aim now is to show that the solution for the case $g_{22} \neq g_{11}$ can be obtained from the solution already developed for the case $g_{22}=g_{11}$, by coordinate transformation. To carry out this 
scheme, we follow Potter and Tuttle [17] and assume that the curves $\eta=$ const, including the boundaries $\Gamma_{1}$ and $\Gamma_{2}$ in the physical plane, are free from sources and sinks. This condition establishes a unique correspondence between the $\xi$-points on each pair of $\eta=$ const lines. In the absence of sources and sinks we must have

$$
\operatorname{div}[\operatorname{grad} \psi(\eta)]=0,
$$

where $\psi(\eta)$ is an arbitrary differentiable function of $\eta$ and, as usual, $\operatorname{grad} \psi(\eta)$ is oriented along the normal to the curve $\eta=$ const. Carrying out the differential operation in (32) and using the following expressions for orthogonal coordinates

$$
|\operatorname{grad} \eta|=\frac{1}{\sqrt{g_{22}}} \text { and } \nabla^{2} \eta=\frac{1}{\sqrt{g}} \frac{\partial}{\partial \eta}\left(\sqrt{\frac{g_{11}}{g_{22}}}\right)
$$

in (32), we obtain

$$
\frac{\partial}{\partial \eta}\left(\ln \sqrt{g_{11} / g_{22}}\right)=-\frac{d^{2} \psi}{d \eta^{2}} / \frac{d \psi}{d \eta} .
$$

Writing $d \psi / d \eta=1 / \nu(\eta)$ and denoting the arbitrary function due to integration by $\ln \mu(\xi)$, we obtain the result

$$
\sqrt{g_{11} / g_{22}}=\mu(\xi) \nu(\eta)=1 / F .
$$

This result shows that for the case of orthogonal coordinates the ratio $g_{11} / g_{22}$ can be taken as a product of the positive functions $\mu(\xi)$ and $\nu(\eta)$. The result in (33) also provides the condition for the two distinct families of orthogonal curves

$$
\xi=\text { const }, \quad \eta=\text { const }
$$

to divide the physical plane in infinitesimal squares [5], as is shown below.

We now introduce new coordinates $\xi^{\prime}(\xi)$ and $\eta^{\prime}(\eta)$ as

$$
\xi^{\prime}=\int \mu(\xi) d \xi, \quad \eta^{\prime}=\int \frac{d \eta}{\nu(\eta)},
$$

then

$$
g_{11}^{\prime}=g_{11} / \mu^{2}, \quad g_{22}^{\prime}=g_{22} \nu^{2},
$$

so that $g_{22}^{\prime}=g_{11}^{\prime}$, i.e., the coordinates $\xi^{\prime}$ and $\eta^{\prime}$ are isothermic.

Defining $P^{\prime}=\ln g_{11}^{\prime}$ and carrying out the transformation (34) in Eq. (11), we have

$$
\frac{\partial}{\partial \xi}\left[\frac{1}{F g_{11}} \frac{\partial}{\partial \xi}\left(F^{2} g_{11}\right)\right]+\frac{\partial}{\partial \eta}\left(\frac{1}{F g_{11}} \frac{\partial g_{11}}{\partial \eta}\right)=\frac{\mu(\xi)}{\nu(\eta)}\left(\frac{\partial^{2} P^{\prime}}{\partial \xi^{\prime 2}}+\frac{\partial^{2} P^{\prime}}{\partial \eta^{\prime 2}}\right)=0 \text {. }
$$

Also, from (7) by using $g_{12}=0$ and by cross differentiation, we have

$$
\frac{\partial}{\partial \xi}\left(\frac{\xi_{x}}{F}\right)+\frac{\partial}{\partial y}\left(\frac{\xi_{y}}{F}\right)=0
$$

which, on using (34), yields

$$
\nabla^{2} \xi^{\prime}=0
$$

From (35) and (36) we conclude that the solution of (11) for the nonisothermic coordinates is the same as the solution of the equation

$$
\frac{\partial^{2} P^{\prime}}{\partial \xi^{\prime 2}}+\frac{\partial^{2} P^{\prime}}{\partial \eta^{\prime 2}}=0
$$


in the isothermic coordinates $\xi^{\prime}, \eta^{\prime}$. In other words, if the solution of (14) is known then the solution of (11) can be obtained by a coordinate transformation $\xi=\phi(\bar{\xi}), \eta=f(\bar{\eta})$ simply by substituting $\phi$ and $f$ in place of $\xi$ and $\eta$ in (19). The salient feature of the preceding analysis is that the solution for the case $g_{22}=g_{11}$ can be used to obtain the solution for the case $g_{22} \neq g_{11}$ by a coordinate transformation in a straightforward noniterative manner.

2.4 Coordinate Transformation. The procedure of transformation from the isothermic coordinate $\xi, \eta$ to the nonisothermic coordinates $\bar{\xi}, \bar{\eta}$ is as follows. On transformation, the covariant metric coefficients transform as

$$
\bar{g}_{i j}=g_{k l} \frac{\partial x^{k}}{\partial \bar{x}^{i}} \frac{\partial x^{l}}{\partial \bar{x}^{j}},
$$

so that, on using the equations $g_{12}=0, g_{22}=g_{11}$, we have

$$
\bar{g}_{11}=\left[(\partial \xi / \partial \bar{\xi})^{2}+(\partial \eta / \partial \bar{\xi})^{2}\right] g_{11}, \quad \bar{g}_{22}=\left[(\partial \xi / \partial \bar{\eta})^{2}+(\partial \eta / \partial \bar{\eta})^{2}\right] g_{11} \text {. }
$$

We now introduce the transformation

$$
\xi=\phi(\bar{\xi}), \quad \eta=f(\bar{\eta}),
$$

where $\phi$ and $f$ are continuously differentiable and satisfy the conditions

$$
\phi\left(\bar{\xi}_{0}\right)=0, f\left(\bar{\eta}_{\beta}\right)=0,
$$

where $\xi=0$ corresponds to $\bar{\xi}=\bar{\xi}_{0}$ and $\eta=0$ corresponds to $\bar{\eta}=\bar{\eta}_{\beta}$. Defining

$$
\lambda=d \phi / d \bar{\xi}, \quad \boldsymbol{\theta}=d f / d \bar{\eta},
$$

we obtain from (37) and (38)

$$
\bar{g}_{22}(\bar{\xi}, \bar{\eta})=\frac{\theta^{2}}{\lambda^{2}} \bar{g}_{11}(\bar{\xi}, \bar{\eta})
$$

The value of $\bar{g}_{22}$ given in (40) must be used in (24) for the evaluation of $x$ and $y$. Further, the prescribed forms in (38) are to be used in place of $\xi$ and $\eta$ in the solution (19). It must be noted that the coefficients $a_{0}, \ldots, d_{n}$, defined in (21), do not depend on the coordinate transformation.

3. Numerical Method of Solution. Based on the formulation of the problem as discussed in the preceding section, we now have a noniterative algebraic computational problem which can be handled in a straightforward manner. Having determined $x(\xi)$ and $y(\xi)$ for both the inner and outer boundaries as described in Section (2.2), we first calculate $\left(g_{11}\right)_{\beta}$ and $\left(g_{11}\right)_{\infty}$ and then $P_{\beta}(\xi)$ and $P_{\infty}(\xi)$, numerically. Based on these distributions, the Fourier coefficients $a_{n}, b_{n}, c_{n}$ and $d_{n}$ are computed by numerical quadrature through the use of (21). Since these values of the coefficients are independent of the spacings between $\eta=$ const lines, the same values are used when a redistribution of $\eta=$ const lines is desired. Substituting the Fourier coefficients in (19) we determine the values of $P(\xi, \eta)$ and hence of $g_{11}(\xi, \eta)$ for the whole annular region. A knowledge of the Fourier coefficients determines $\alpha(\xi, \eta)$ through (23) and so also the Cartesian coordinates through (24) by numerical quadrature. The value of $\rho_{0}$ from (28) and (29) by numerical quadrature determines $\eta_{\infty}$ through (30) and so of $\bar{K}$ in (20). 


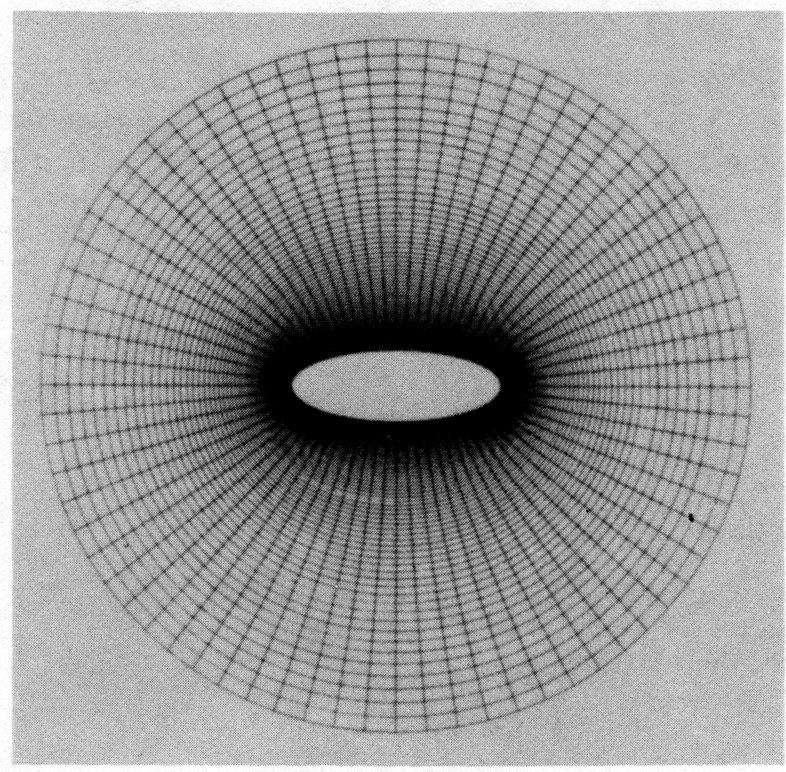

\section{Figure 3}

Confocal ellipses. Semimajor axes 1.48, 5.0, and semiminor axes 0.5, 4.802 respectively. Only $38 \eta=$ const lines shown for detail

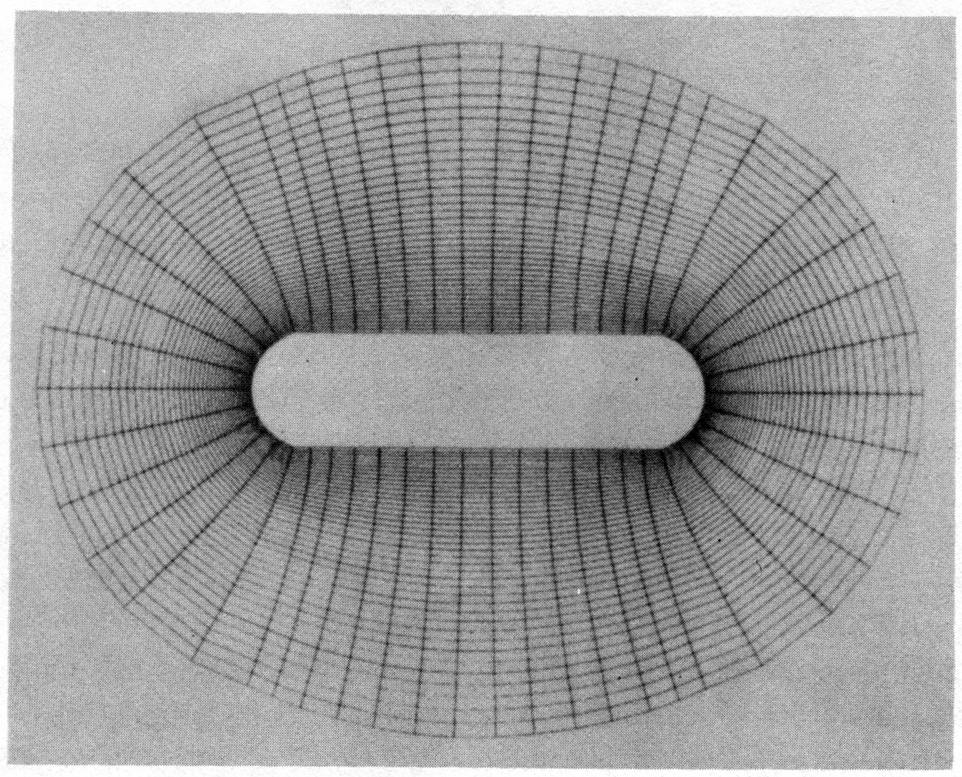

FIGURE 4

A blunt body section with elliptical outer boundary 


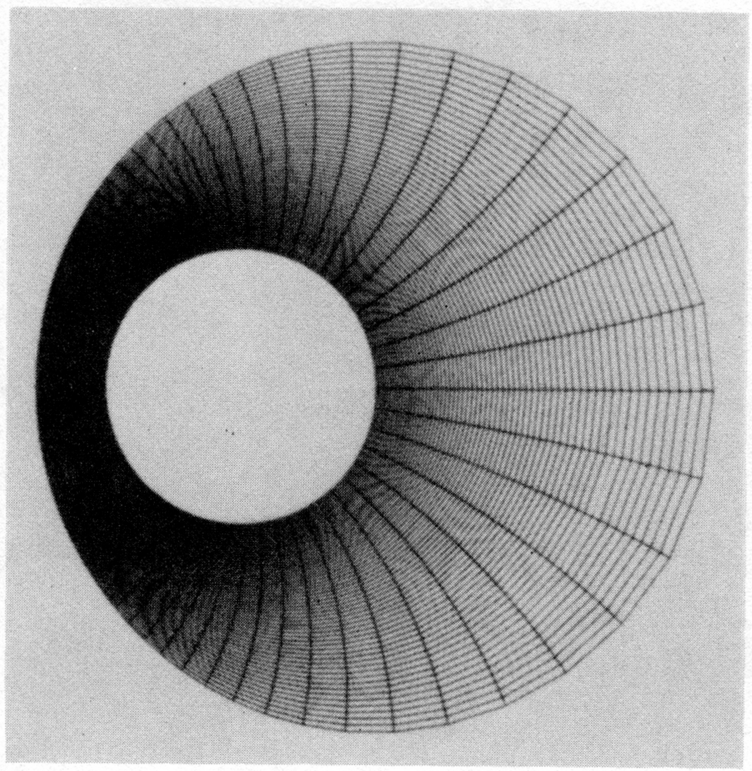

Figure 5

Nonconcentric circles: $r_{S}=1, r_{L}=2.5, z_{S}=(0,0), z_{L}=(1,0)$

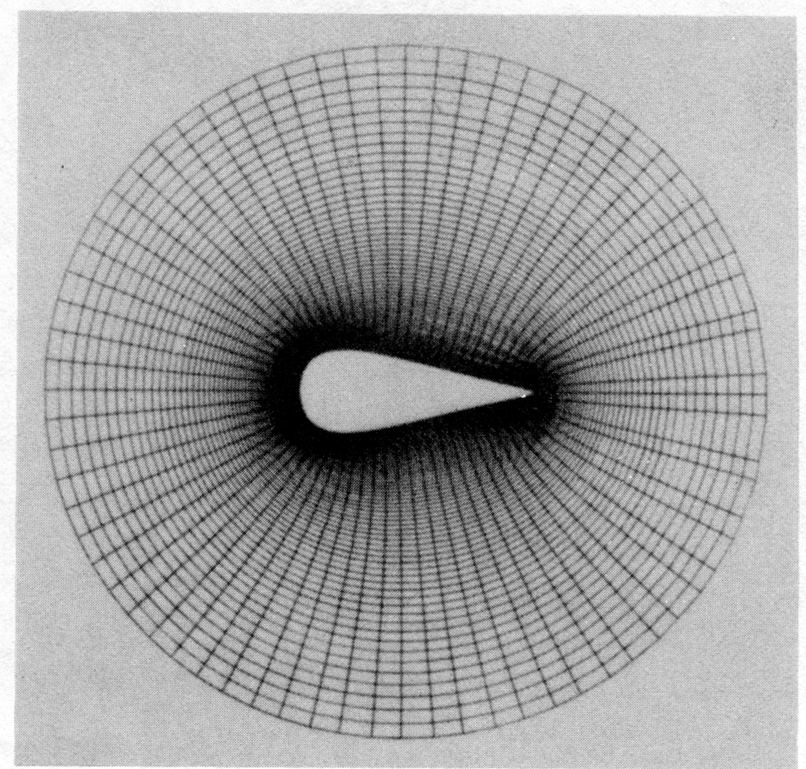

FIGURE 6

Joukowsky's airfoil with slightly rounded trailing edge 


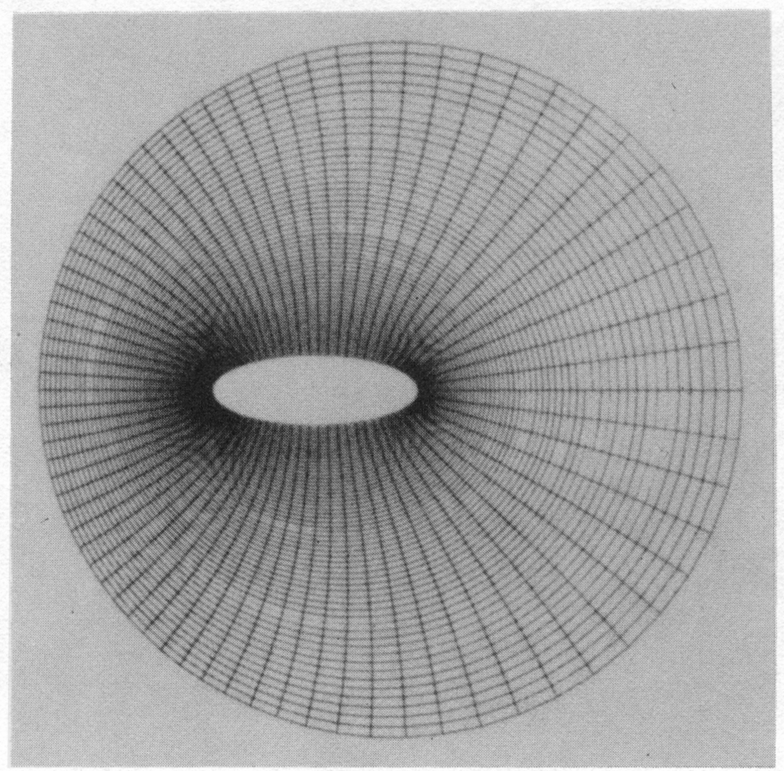

FIGURE 7

Nonconcentric ellipses. Size data same as in Figure 3. $z_{S}=(0,0), z_{L}=(1,0)$.

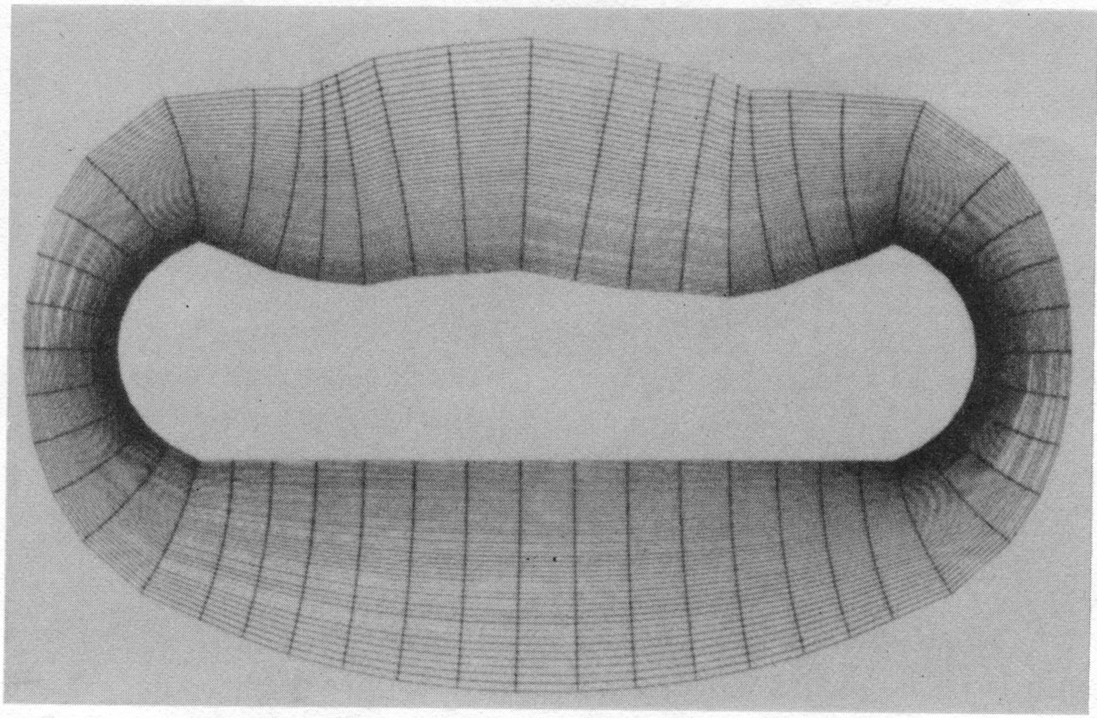

\section{FIGURE 8}

Generated coordinates for a body having convex, concave and straight portions. Placement of outer boundary is decided by the radius of the osculating circles of the concave portions. 
A computer program, [22], with the option of redistributing the coordinate lines in any desired manner (subject to the constraints on $\bar{\xi}$ discussed in Section 2.2) has been written and used to generate the orthogonal coordinates for various annular regions as shown in Figures 3-8. All these runs are for the case of nonisothermic coordinates. For these example problems we have chosen the following forms of the functions $\phi$ and $f$ mentioned in (38).

$$
\begin{gathered}
\phi(\bar{\xi})=\frac{2 \pi\left(\bar{\xi}-\bar{\xi}_{0}\right)}{\bar{\xi}_{m}-\bar{\xi}_{0}} \\
f(\eta)=\frac{\eta_{\infty}\left(\bar{\eta}-\bar{\eta}_{\beta}\right)}{\bar{\eta}_{\infty}-\bar{\eta}_{\beta}} \cdot \frac{K^{\left(\bar{\eta}-\bar{\eta}_{\beta}\right)}}{K^{\left(\bar{\eta}_{\infty}-\bar{\eta}_{\beta}\right)}}
\end{gathered}
$$

so that $\lambda, \theta$ defined in (39) are

$$
\lambda=\frac{2 \pi}{\bar{\xi}_{m}-\bar{\xi}_{0}}, \quad \theta=\frac{\eta_{\infty}}{\bar{\eta}_{\infty}-\bar{\eta}_{\beta}}\left[1+\left(\bar{\eta}-\bar{\eta}_{\beta}\right) \ln K\right] \frac{K^{\left(\bar{\eta}-\bar{\eta}_{\beta}\right)}}{K^{\left(\bar{\eta}_{\infty}-\bar{\eta}_{\beta}\right)}},
$$

where $K>1$ is an arbitrary constant; $\bar{\xi}=\bar{\xi}_{m}$ and $\bar{\eta}=\bar{\eta}_{\infty}$ correspond, respectively, to $\xi=2 \pi$ and $\eta=\eta_{\infty}$. We treat $\bar{\xi}$ and $\bar{\eta}$ as integers so that

$$
\bar{\xi}_{0}=1, \quad \bar{\xi}_{m}=\text { IMAX, } \quad \bar{\eta}_{\beta}=1, \quad \bar{\eta}_{\infty}=\text { JMAX. }
$$

Since $\eta_{\infty}$ is known from (30), hence, by specifying the numerical values of $K$ and JMAX, we can create a desired mesh control in the $\eta$ direction. The value of $K$ between $1 \cdot 05$ and $1 \cdot 1$ is quite sufficient, [23], in having a very fine grid spacing near the inner boundary.

The number of terms to be retained in the series (19) is usually small for convex inner and outer boundaries, though we have retained (IMAX - 1)/2 number of coefficients in each computation. This number is the optimum number of terms in a discrete Fourier series having IMAX number of points in one period, [12]. The average computer time for the complete solution on the UNIVAC 1100/80 for $\operatorname{IMAX}=73, \mathrm{JMAX}=60$ field is about 2.75 minutes.

4. Conclusions. The Gaussian equation for a plane under the condition of orthogonality of coordinates has been used to form a basis for generating orthogonal coordinates in plane annular regions. The resulting method is noniterative and requires only the numerical evaluation of integrals by quadratures and summation of finite (truncated) Fourier series. The method works very effectively for smooth and convex boundaries of any shape or orientation. However, in the case of concave inner boundaries, the outer boundary has to be placed at such a distance so as to avoid intersecting normals, [7]. Further, sharp turns and corners are not admissible and have to be rounded to avoid singularities in the metric data.

Acknowledgement. A computer program for the analysis reported here was developed by R. A. Weed. 
1. A. A. Amsden \& C. W. Hirt, "A simple scheme for generating general curvilinear grids," $J$. Comput. Phys., v. 11, 1973, pp. 348-359.

2. W. D. BARField, "An optimal mesh generator for Lagrangian hydrodynamic calculations in two space dimensions," J. Comput. Phys., v. 6, 1970, pp. 417-429.

3. J. BuRbEA, "A numerical determination of the modulus of doubly connected domains by using the Bergman curvature," Math. Comp., v. 25, 1971, pp. 743-756.

4. W. H. CHU, "Development of a general finite difference approximation for a general domain, Part I: Machine transformation," J. Comput. Phys., v. 8, 1971, pp. 392-408.

5. A. COHEN, An Introduction to the Lie Theory of One-Parameter Groups, Stechert, New York, 1931.

6. R. T. DAvis, Numerical Methods for Coordinate Generation Based on Schwarz-Christoffel Transformation, AIAA Computational Fluid Dynamics Conference, Paper No. 79-1463, 1979.

7. P. R. EISEman, "A coordinate system for a viscous transonic cascade analysis," J. Comput. Phys., v. 26, 1978, pp. 307-338.

8. L. P. Eisenhart, Riemannian Geometry, Princeton Univ. Press, Princeton, N. J., 1926.

9. D. GAIER, Determination of Conformal Modulus of Ring Domains and Quadrilaterals, Lecture Notes in Math., vol. 399, Springer-Verlag, Berlin, 1974.

10. S. K. Godunov \& G. P. Prokopov, "The use of moving meshes in gas dynamics computations," USSR Comput. Math. and Math. Phys., v. 12, 1972, pp. 182-195.

11. G. M. Goluzin, Geometric Theory of Functions of a Complex Variable, Transl. Math. Monos., vol. 26, Amer. Math. Soc., Providence, R. I., 1969.

12. F. B. Hildebrand, Introduction to Numerical Analysis, McGraw-Hill, New York, 1956.

13. H. KoBer, Dictionary of Conformal Representations, Dover, New York, 1952.

14. J. F. Middlecoff \& P. D. Thomas, Direct Control of the Grid Point Distribution in Meshes Generated by Elliptic Equations, AIAA Computational Fluid Dynamics Conference, Paper No. 79-1462, 1979.

15. C. D. Mobley \& R. J. Stewart, "On the numerical generation of boundary-fitted orthogonal curvilinear coordinate systems," J. Comput. Phys., v. 34, 1980, pp. 124-135.

16. S. B. POPE, "The calculation of turbulent recirculating flows in general orthogonal coordinates," $J$. Comput. Phys., v. 26, 1978, pp. 197-217.

17. D. E. PotTer \& G. H. Tutrle, “The construction of discrete orthogonal coordinates," J. Comput. Phys., v. 13, 1973, p. 483.

18. M. K. RichaRdSON \& H. B. Wilson, "A numerical method for the conformal mapping of finite doubly connected regions," Developments in Theoretical and Applied Mechanics, Vol. 3, Ed., W. A. Shaw, Plenum Press, 1967.

19. G. STARIUS, "Constructing orthogonal curvilinear meshes by solving initial-value problems," Numer. Math., v. 28, 1977, pp. 25-48.

20. J. F. Thompson, F. C. Thames \& C. W. MASTin, "Automatic numerical generation of body-fitted curvilinear coordinate system for field containing any number of arbitrary two-dimensional bodies," $J$. Comput. Phys., v. 15, 1974, pp. 299-319.

21. J. F. Thompson, F. C. Thames \& C. W. Mastin, “'TOMCAT'-A code for numerical generation of boundary-fitted curvilinear coordinate system on fields containing any number of arbitrary twodimensional bodies," J. Comput. Phys., v. 24, 1977, pp. 274-302.

22. Z. U. A. WARSI, R. A. WEED \& J. F. ThOMPSON, Numerical Generation of Two-Dimensional Orthogonal Coordinates in an Euclidean Space, Engineering and Experimental Research Station, Mississippi State University, Rep. No. MSSU-EIRS-ASE-80-3, June 1980.

23. Z. U. A. WARSI \& J. F. Thompson, Machine Solutions of Partial Differential Equations in the Numerically Generated Coordinate Systems, Engineering and Experimental Research Station, Mississippi State University, Rep. No. MSSU-EIRS-ASE-77-1, August 1976.

24. A. J. Winslow, "Numerical solution of the quasi-linear Poisson equation in a non-uniform triangular mesh," J. Comput. Phys., v. 2, 1966, pp. 149-172. 